\title{
Combining Digital Archives Content with Serious Game Approach to Create a Gamified Learning Experience
}

\author{
Deng-Teng Shih a, Chan Li Lin ${ }^{b}$, Ching-Yueh Tseng c* \\ a Dept. of Digital Multimedia Design, China University of Technology, Taipei, Taiwan - leonshihshih0907@cute.edu.tw \\ ${ }^{b}$ Dept. of Cultural and Creative Industries Management, National Taipei University of Education, Taipei, Taiwan - \\ chanli@tea.ntue.edu.tw \\ cDept. of Digital Multimedia Design, China University of Technology, Taipei, Taiwan - jingyueh@cute.edu.tw
}

KEY WORDS: Digital Archives, Gamification, Serious Game, Hukou Old Street

\begin{abstract}
:
This paper presents an interdisciplinary to develop content-aware application that combines game with learning on specific categories of digital archives. The employment of content-oriented game enhances the gamification and efficacy of learning in culture education on architectures and history of Hsinchu County, Taiwan. The gamified form of the application is used as a backbone to support and provide a strong stimulation to engage users in learning art and culture, therefore this research is implementing under the goal of "The Digital ARt / ARchitecture Project".
\end{abstract}

The purpose of the abovementioned project is to develop interactive serious game approaches and applications for Hsinchu County historical archives and architectures. Therefore, we present two applications, "3D AR for Hukou Old " and "Hsinchu County History Museum AR Tour" which are in form of augmented reality (AR). By using AR imaging techniques to blend real object and virtual content, the users can immerse in virtual exhibitions of Hukou Old Street and Hsinchu County History Museum, and to learn in ubiquitous computing environment.

This paper proposes a content system that includes tools and materials used to create representations of digitized cultural archives including historical artifacts, documents, customs, religion, and architectures. The Digital ARt / ARchitecture Project is based on the concept of serious game and consists of three aspects: content creation, target management, and AR presentation. The project focuses on developing a proper approach to serve as an interactive game, and to offer a learning opportunity for appreciating historic architectures by playing AR cards. Furthermore, the card game aims to provide multi-faceted understanding and learning experience to help user learning through 3D objects, hyperlinked web data, and the manipulation of learning mode, and then effectively developing their learning levels on cultural and historical archives in Hsinchu County.

\section{INSTRODUCTION}

\subsection{General Instructions}

There are two experimental applications are included in the Digital ARt / ARchitecture Project, they are "3D AR for Hukou Old Street" and "Hsinchu County History Museum AR Tour". These two applications aim to convert the selected material of local culture and history them into AR entertainments. As we know, global culture has shifted aside local culture within the society and made it less attractive in comparison with modern culture. By applying game design, the Digital ARt / ARchitecture Project develops content-aware applications / games that combine gamification experience with local culture learning when the users are playing the AR game. Users are hoped to feel a different sensation when they are playing these games. The gamified form of designed content is used as a backbone to support and provide a strong stimulation to engage users to comprehend information of history and architecture in Hsinchu County. The project transforms artistic cultural heritages of Hsinchu County into many types of AR content, such as in page video, full screen video, website hyperlink, text, image, animation, 3D objects, and so forth. By playing "3D AR for Hukou Old Street", an AR table card game; the users will immerse in a virtual scene of Hukou Old Street and learn the beauty of Hakka culture and historic architectures in ubiquitous computing environment. Meanwhile, users can experience an add-on exhibition through the device screen that is served as AR application of Hsinchu County History Museum without Augmented Reality (AR) technology extends Virtual Reality (VR) performances by blending real and virtual elements into real scenes, and to enrich the visual object with a non-artificial view of real scenes. AR technology has already made vivid impact on many domains. This paper focus on how AR techniques can offer a great solution of enabling visualization of 3D models of cultural architectures to be applied in art education for children and to be used to enhance user's cognitive skills.

This research investigates the feasibility that interactive AR game is used to support mobile learning. Since we believe the interactive experience and information display in the augmented world can stimulate AR game user's sense of participation and excitement. Meanwhile, the serious games represented in this paper aims to function AR features on the application of smart traveling and virtual exhibition. Therefore, we present "3D AR for Hukou Old Street" and "Hsinchu -County History Museum AR Tour".

* Corresponding author 


\subsection{Research Motivation}

In the Digital Hukou Project "3D AR for Hukou County Adventure for Hukou Treasures", local art history in this case, local culture $\sim$ is being converted into an AR card game. Global culture has shifted aside local culture within the society and made it less attractive in comparison with modern foreign culture. By using game design, the Digital Hukou Project aims to develop a content-aware application/ game that combines gamification experience with local culture learning when the users are playing the game. readers are hoped to feel a different sensation when they are playing this game. The gamified form of digital content is used as a backbone to support and provide a strong stimulation to engage users to comprehend and enjoy art/architecture information of Hukou Old Street. The project transforms artistic cultural heritage of Hukou Old Street into content of AR application. By playing AR table cards, the users will immerse in a virtual scene of Hukou Old Street and learn the beauty of Hakka culture and historic architectures in ubiquitous computing environment.

Augmented Reality (AR) technology extends Virtual Reality (VR) performances by blending real and virtual elements into real scenes, and to enrich the visual object with a non-artificial view of real scenes. AR technology has already made vivid impact on many domains. This paper focus on how AR techniques can offer a great solution of enabling visualization of 3D models of cultural architectures to be applied in art education for children and to be used to enhance user's cognitive skills.

\subsection{Research Background}

Hukou Old Street had been once prospered and is now declined because of the changing of railroad transportation. Located in Hukou Township, Hsinchu County, and the old street is a long street occupied by two-story redbrick buildings that were built around 1920 and are probably the most complete Japanese-era shop-houses in Taiwan. Therefore, the project focus on developing a proper approach to serve as interactive game, and offer a learning opportunity on Hukou Old Street through playing cards to appreciate Hukou historic architectures.

When the direction of information technology gradually being taken seriously into the expected development of education model, along with the boom in hardware and software of mobile devices, constructing a multimedia and interactive digital learning environment that provides more learning opportunities and quality optimisation to enhance cognitive and affective skills. Therefore, the knowledge/ information of cultural architectures and historical archive is transformed into real-time rendering of high-end video, synchronised transmission of voice, text, images, objects, and other learning content in the AR card game.

There are many related researches published recently. Kou and Jeng (2008) collected and classified a number of papers on architecture subject that are related to the augmented reality research. They collected 121 papers from journals published between 1968 and 2006 and categorized into "Outdoors tour of architecture and urban", "exhibition and learning environment", "Design and practical project", "interior and ancillary equipment". They also indicated number of study on "Outdoors tour of architecture and urban" is only half to a quarter of the other three, and speculated that augmented reality technology is facing a dilemma on studying and developing the application of outdoors tour.

\subsection{Objective}

The present project for building a virtual scene of cultural architectures and a virtual exhibition of historical archives enables user, player, tourists, visitors to bring magnificent historical art and architectures closer to see and use for the purpose of interactive appreciating and learning. The solution of this project makes cultural architectures and historical archives portable and interactive, and furthermore, to transform viewers into players to interact with $\mathrm{AR}$ content in an intuitive and exciting manner. Therefore, this research aims to evaluate the current development of game-based learning and presents an overview in achieving interoperability between playing and learning/appreciating.

Lehner and Nosekabel (2002) defined mobile learning as "A service that gives the student general information and educational content in electronic form, which help the learner to acquire new knowledge at any time and anywhere."

We believe mobile devices can ease restrictions regarding the location of learning using features of AR technology and serious game. Since game is an attractive and effective medium to interpret solid knowledge into pleasure information through designed images, music, sound effects, and missions. By applying game as a medium, it is hoped that the process of reintroducing Hukou historical buildings and exhibition of Hsinchu County History Museum can be a more easier and fun way for user to absorb the designated information.

\subsection{Methodology}

Augmented Reality lets users interact with the real world and virtual objects simultaneously. 3D models are overlaid on the real world as seen through device camera making them appear to part of surrounding real environment.

As we know that virtual reality seeks to replace the real world, but augmented reality aims to subjoin information in the real world. The users in the virtual reality are deliberately separated from real world, while some users immerse the coexisting world between the virtual and real in augmented reality. While other AR applications focus on augmenting the real world and objects with a lot of digital information, the applications presented in this research explore game-based learning in real-world contexts that are intentionally augmented using digital information from mobile devices.

To stimulate user's interest to learn about local culture by playing card game, this project uses AR technology to make game more attractive and interesting to introduce Hukou historical heritages. This project applies game design method to develop the concept of the serious game on certain subject of the past Hukou life. We had built 27 pieces of 3D buildings and created more than 10 game characters for this Hukou card game and the AR application.

In this game, the figures possess special attributes served as crucial elements allow users to recognize the figures as Hakka people. On the other hand, Hukou Old Street is classical in appearance and is accepted as one of representative settlements of Japanese-era architecture. The traditional looks of Hukou are preserved well after several renovation projects. However, these buildings not only differs from each other to show their own individual feature, but also decorates with relief motifs of Chinese-style feature such as dragons, cranes, and immortals to 
declare it origins. Hence this project will present $273 \mathrm{D}$ buildings to serve as the AR image with its paired AR card.

There are two elements differ Augmented Reality from Virtual Reality greatly. They are virtual objects in the real world and complex interaction between virtual and reality. Since the real world is too complex and delicate to be simulated, therefore AR application aims to provide overlaying digital information to the real world. Because of these advantage and features, AR technology had been widely used in applications of mobile learning, smart traveling, and exhibition tour.

Sung, Chang, Lee \& Yu (2008) published a research on how the influence of using mobile tour guide application is to the museum visitors. The paper came to a result that the museum tour applying mobile device could improve the concentration of museum visitors, and to stimulate visitor's curiosity and interest on exhibition items.

As mention above, for verifying the theory addressed in this paper, we developed two AR prototype games, "3D AR for Hukou Old Street" and "Hsinchu County History Museum AR Tour", for the Digital ARt / ARchitecture Project. The further information about these two AR games will be addressed in the following sections.

\section{3D AR FOR HUKOU OLD STREET}

\subsection{Game synopsis}

"3D AR for Hukou Old Street Adventure of Hukou Treasures" is a game designed to help users/players to grasp a concept of cultural treasures and to absorb the educational information on Hukou history. The Hukou "treasures" should be defined as a cultural concept but to be designated as any specific person or thing. This game brings the beauty of Hukou local culture and traditional architectures to the palms of players. However, the purpose for designing this game is to stimulate players' motivation to pay a visit to nowadays Hukou Old Street and to taste the cultural atmosphere in person.

\subsection{Game design}

These games offer new educational opportunities on technology-enabled learning: the technology supports ubiquitous explorations and learning situated in the natural context: their and museum.

The software that enables this technology combines a simulation engine with features that afford elements of game play-participant roles, goals, feedback, and challenging puzzles - to create games that are truly immersive.

There are 8 main characters in the game; they individually represent the Hakka ethnic, Taiwanese ethnic, aborigine, modern traveller, and catholic priest. These game roles are all in vivid characteristic designs, no matter on clothing, hairstyle, or personality. This game feature aims to provide players with multiple options to play self-chosen roles to immerse in the Hakka society and to experience the story of the Hukou Old Street. Furthermore, the game world is mixture by the past and the present, player immerse in the Hukou historical heritages and nowadays scenes to appreciate the beauty of Hukou culture.

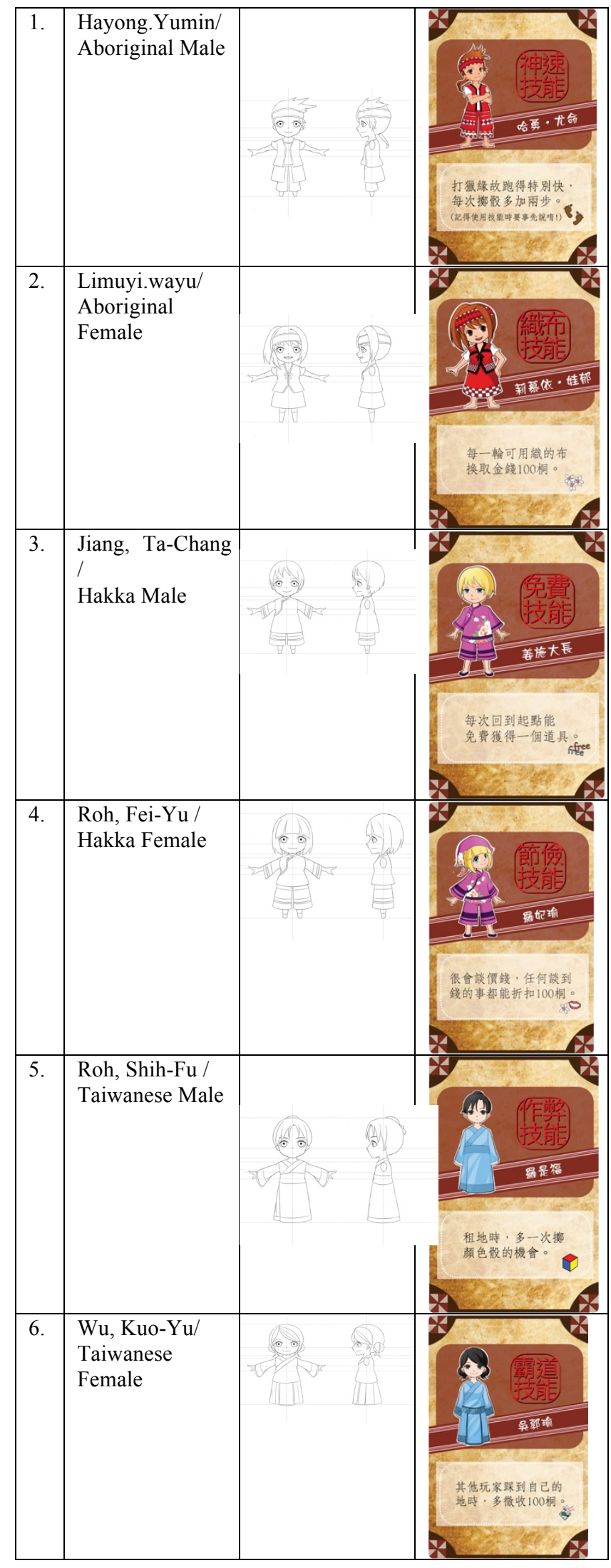

\begin{tabular}{|l|l|l|l|}
\hline No & $\begin{array}{l}\text { Name / } \\
\text { Ethnic/ } \\
\text { Occupation }\end{array}$ & $\begin{array}{l}\text { Character } \\
\text { Design }\end{array}$ & $\begin{array}{l}\text { Card } \\
\text { Design }\end{array}$ \\
\hline
\end{tabular}




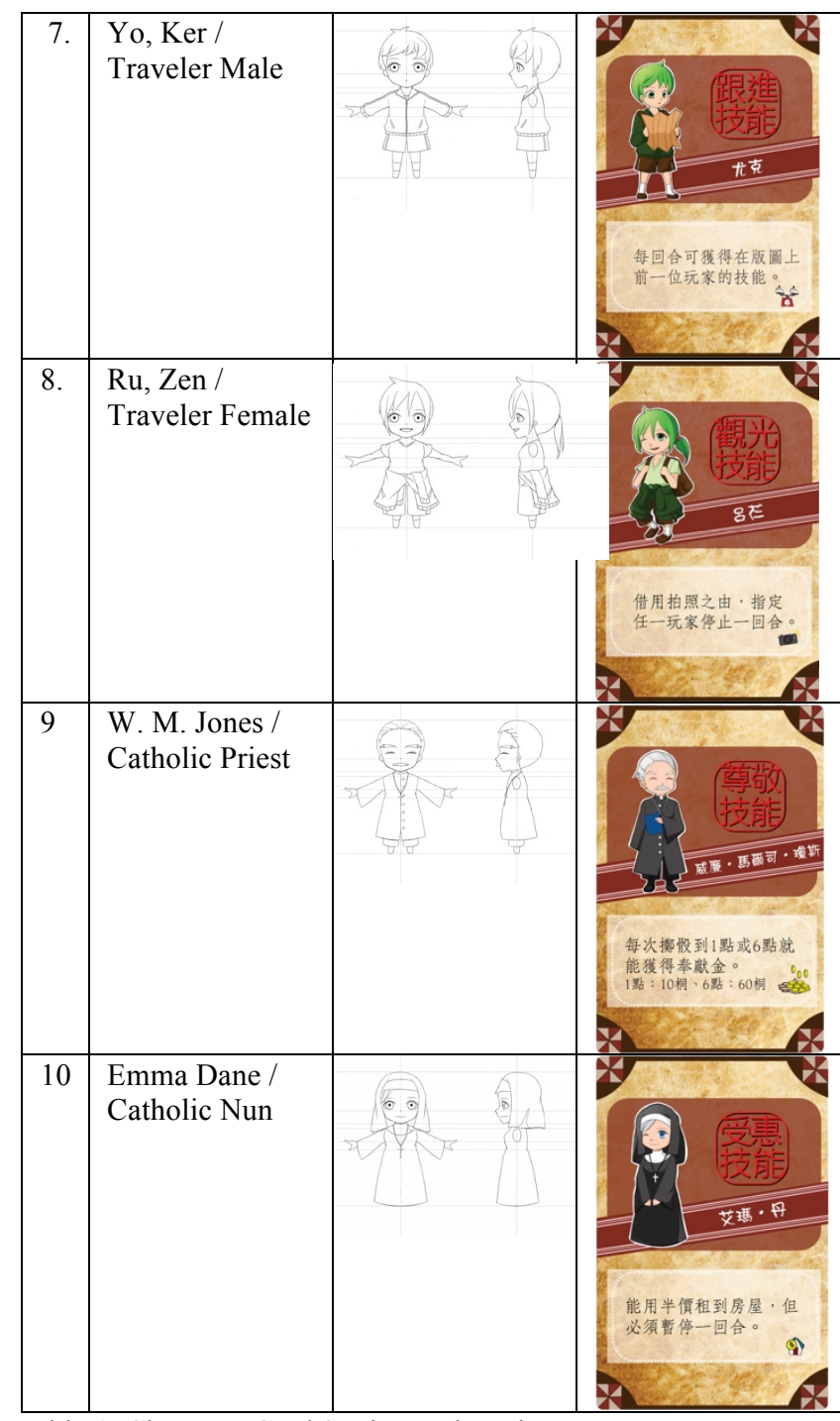

Table 1. Character Card Setting and Design

\begin{tabular}{|l|l|l|l|}
\hline No. & \multicolumn{1}{|c|}{ Name } & \multicolumn{2}{|c|}{ Description } \\
\hline 1. & $\begin{array}{l}\text { The Feast of } \\
\text { Spring Festival } \\
\sim \text { Event } 1\end{array}$ & $\begin{array}{l}\text { To attend the } \\
\text { feast, and to } \\
\text { make local } \\
\text { acquaintance } \\
\text { with } \\
\text { residences. }\end{array}$ \\
\hline 2. & $\begin{array}{l}\text { The Feast of } \\
\text { Spring Festival } \\
\sim \text { Event 2 }\end{array}$ & $\begin{array}{l}\text { Intruding into } \\
\text { the feast in } \\
\text { progress, player } \\
\text { turns away from } \\
\text { the activity. }\end{array}$ \\
\end{tabular}

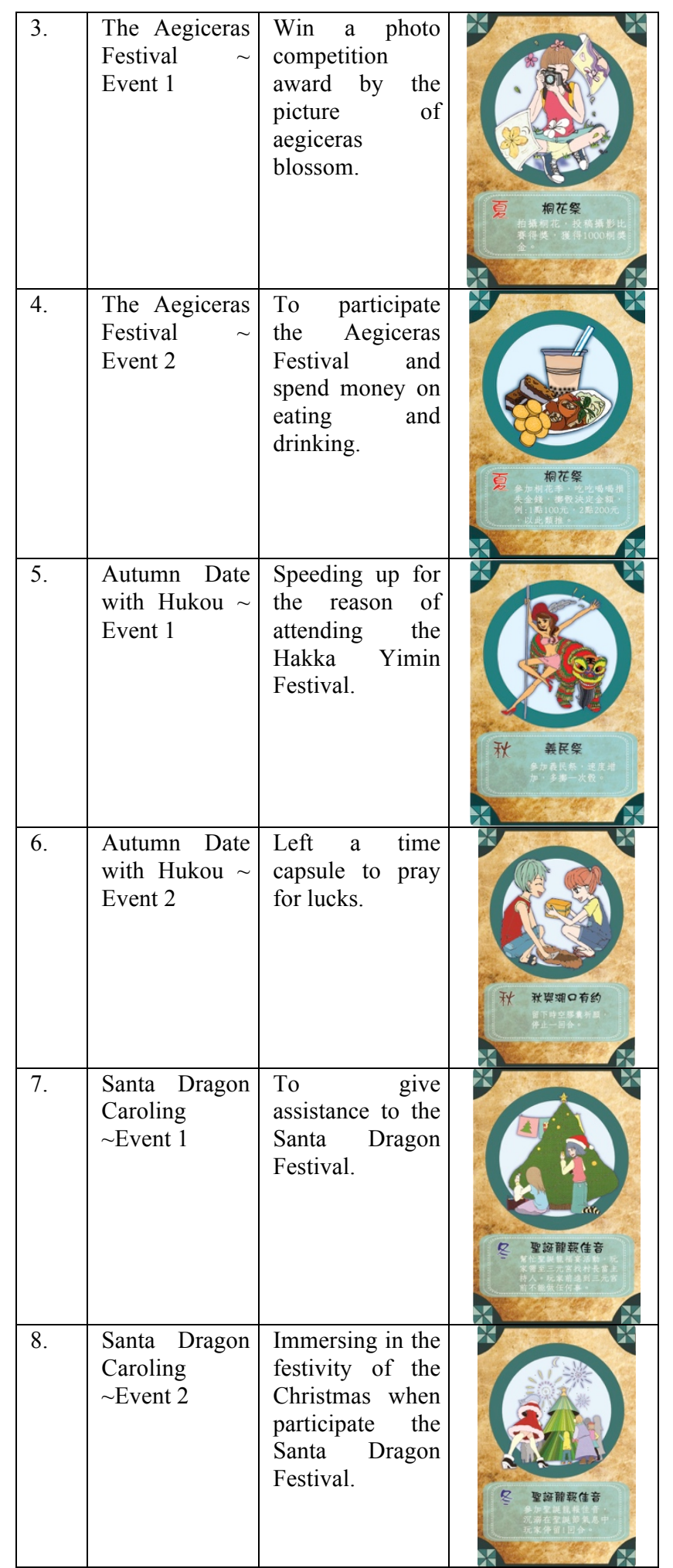

Table 2. Four Seasons Event Card Setting

There are also several sets of Property Card, Chance Card, Fate Card, and Trap Card serve as board game items for players (playing visitor roles of the past Hukou Old Street) to collect, invest. Take the Property Card for example, there are 42 cards in total represent the real estates is located in the Hukou Old Street, and serve as 3D Building Card (front side) and Information Card (rear side) for AR Application to emerge realistic $3 \mathrm{D}$ buildings and Hukou old building information when the card is scanned by users. 


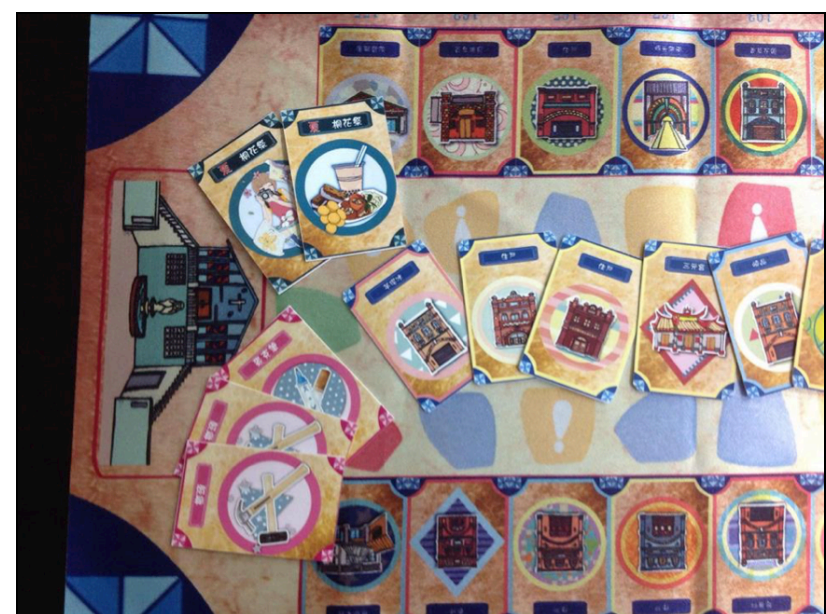

Figure 1. Game board and cards of "3D AR for Hukou Old Street"

\subsection{Game development}

For the reason that player is endowed capability to use "Aegiceras Currency" to purchase personal property in the game, such as building, or game props. Therefore, this project also designs "Building Card" and "Props Card" to act as the ownership of estates and game objects. The building cards can serve as the image markers for AR application to scan to execute augmented imaging function on device and to meet the educational purpose. The developed result is likely to be as seen below:

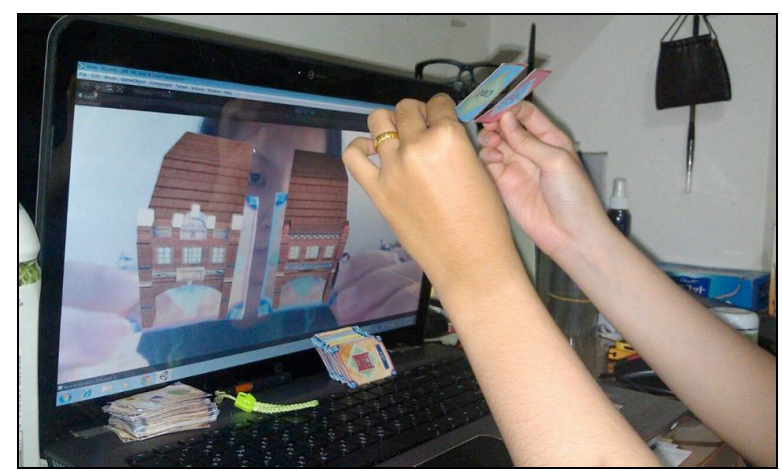

Figure 2. AR card (right) and AR imaging (left)

In this project, the Autodesk $3 \mathrm{ds}$ Max, that enables designers to focus simply on creation, is used to make all three dimensional characters and architectures in this AR able game. For modeling game characters and historical architectures, we conducted field investigations and developed the character settings to use as the $3 \mathrm{D}$ characters design guideline and to meet the standard of cultural digital archive. According to the research result and game design proposal comes after several field works, the project team can then design game cards and 3D objects to use as the content of both the AR application and the table game, as seen in the picture below:

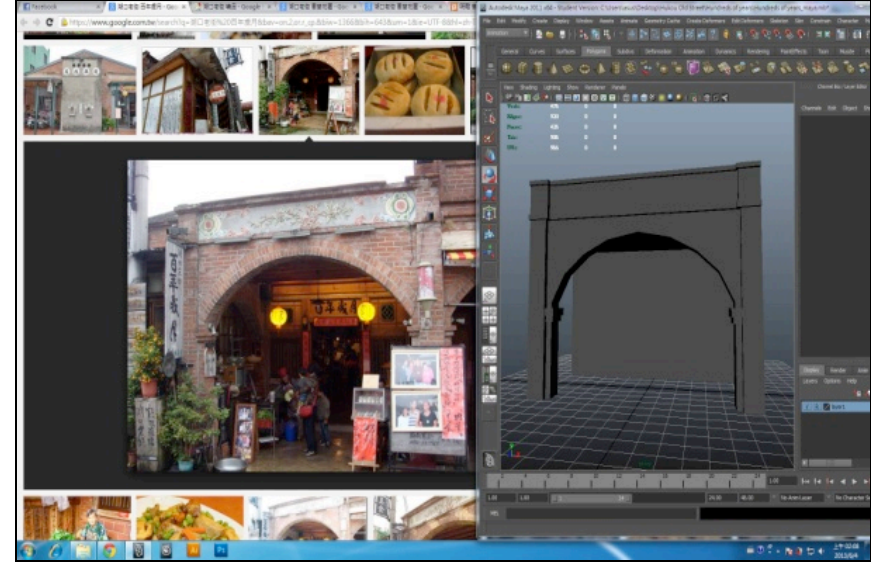

Figure 3. Modeling in 3ds Max (right) and building reference materials (left)

This project proposes a content system that includes tools and materials used to create representations of digitized cultural architectures of Hukou Old Street. The development of AR contents consists of three aspects: content creation, target management, and AR presentation as shown in fig. 4.

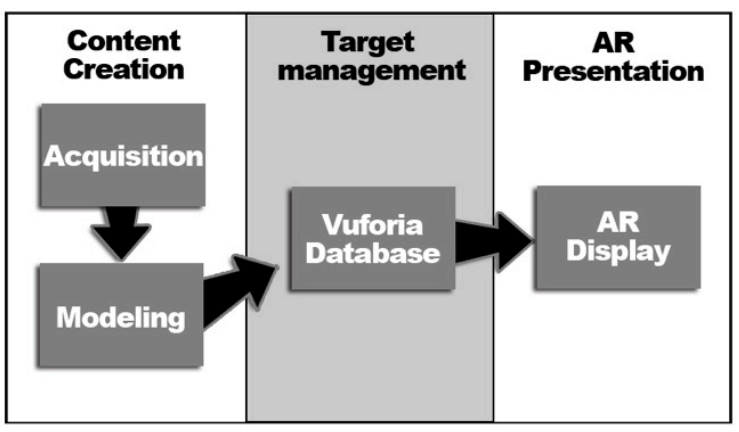

Figure 4. The structure of developing AR contents

3D models of architectures are stored in the Target Database offered by Vuforia SDK-based AR application. With Vuforia software platform, 3D objects come to life with interactivity through $3 \mathrm{D}$ graphics to mobile device and individually show on the corresponding AR card.

\subsection{Result}

Through webcam or mobile device's camera, the front side, which is illustrated with graphic appearance of architecture, will function as AR Target to show corresponding 3D model. Meanwhile, the rear side, which is illustrated in form of doorplate, is design to show the description of details of the specific architecture. This is an import function designated to provide further information for who interested to discover more on any historical architecture. 


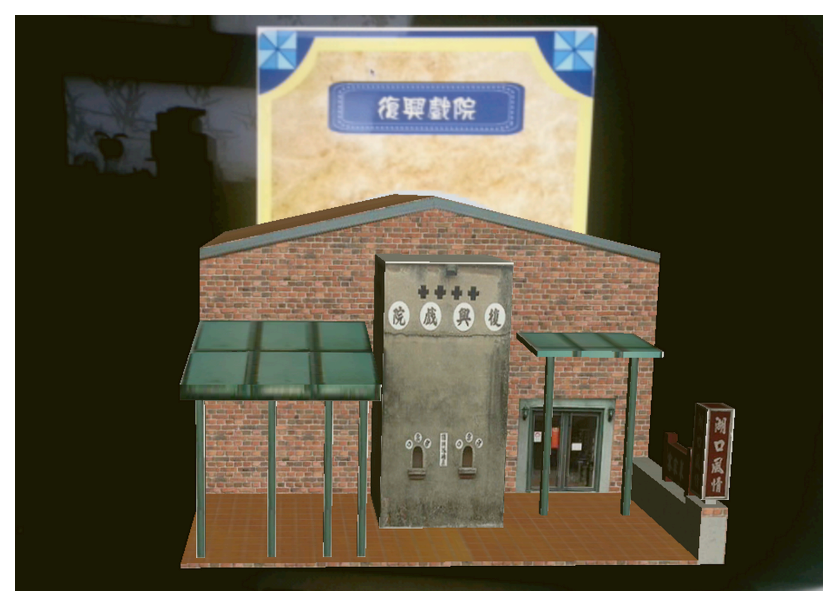

Figure 5. Scanning the front side of the board game card to reveal corresponding $3 \mathrm{D}$ building on the device screen

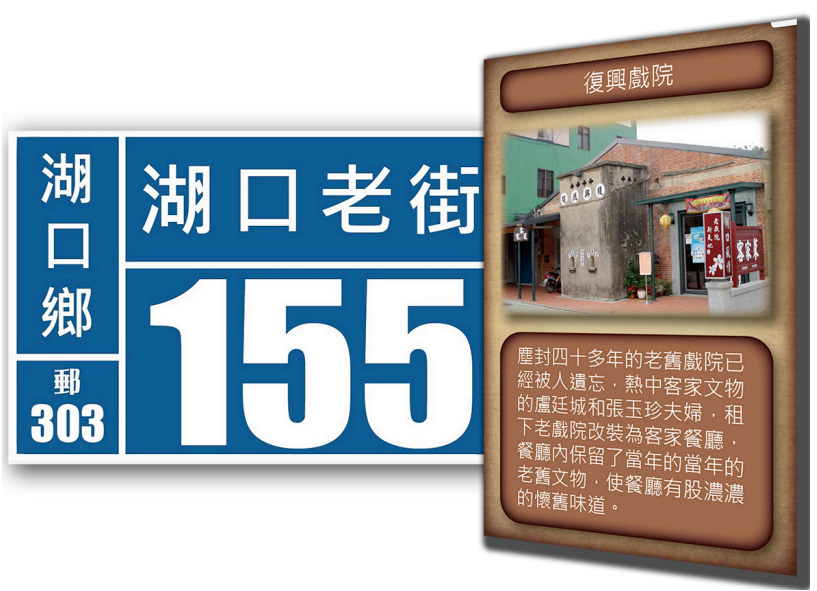

Figure 6. Scanning the door plate (left, the rear side of the board game card) to show further information about the building (right)

"3D AR for Hukou Old Street Adventure of Hukou Treasures" is a game designed to help users/players to grasp a concept of cultural treasures and to absorb the educational information on Hukou history. The Hukou "treasures" should be defined as a cultural concept but to be designated as any specific person or thing. This game brings the beauty of Hukou local culture and traditional architectures to the palms of players. However, the purpose for designing this game is to stimulate players' motivation to pay a visit to nowadays Hukou Old Street and to taste the cultural atmosphere in person.

\section{HSINCHU COUNTY HISTORY MUSEUM AR TOUR}

\subsection{About the Museum and the application}

Hsinchu County History Museum was found in 2004 and was opened formally in 2012. The main function of the museum is to collect and exhibit official archives and civil history records; the museum's collection included old photos, documentary films, old maps, daily supplies, land contracts, excavated materials, religious items, as well as the miniatures of the old stations. The museum organised the permanent exhibition with the solid concept of "Back to the old days", people are all welcome to access and to appreciate the history of Hsinchu County. Besides the exhibition function, the museum was also designed to express a kind of internal spirit for searching the common memory and historical feeling of Hakka People.
However, because construction of the museum was executed under low water of finance condition. The main content of exhibition space still remains unchanged, even if it has been three years from the exhibition room was opened. It means that even though the museum collects rich archive of historical documents, manuscripts, architecture maps, and cultural relics, but there is no space and fund can support to organise an exhibition to display these precious collections.

Therefore, the Digital ARt / ARchitecture Project carried out an experiment idea, that is to apply AR technology on creating a value added virtual exhibition. The innovative exhibition is an AR exhibition grounds on experience of developing "3D AR for Hukou Old Street " but seeks to bring an outdoors tour application into the museum exhibition space. This AR application is named "Hsinchu County History Museum AR Tour" and aims to provide a virtual exhibition on the device screen. There are 27 artefacts in the exhibition room designated as markerless AR tracking objects of the application, and also function as individual new exhibition artefacts to be combined into a complementary exhibition that overlaps the original one.

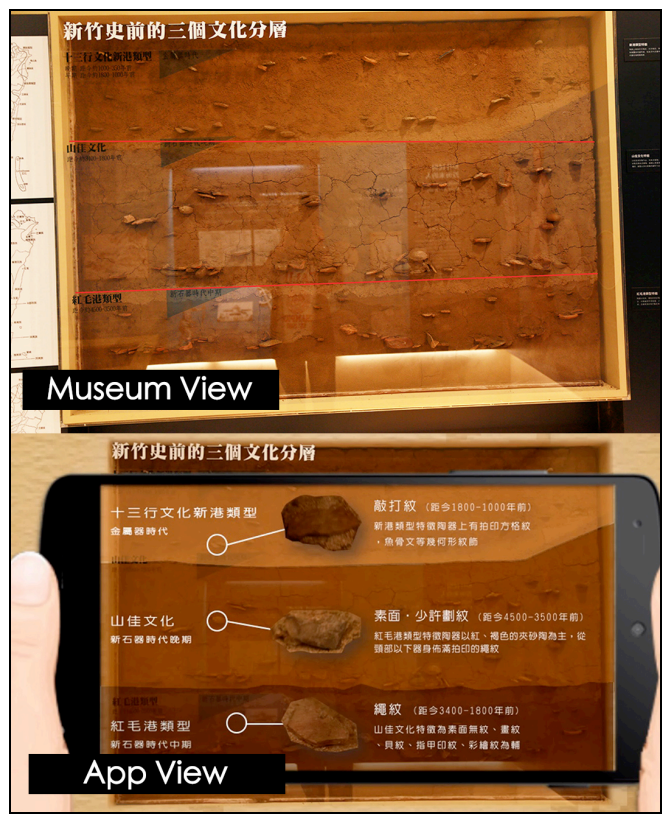

Figure 7. The original exhibition item (above) and the further information showing on the device after scanned (below)

\subsection{Design Idea}

Delivering museum tours digitally is currently a popular method for museum education and for stimulation of visitors' enthusiasm and interest. Visitor / players conduct their visits to the Hsinchu County History Museum with everyone carrying a mobile device can enables them to scan markerless exhibition items through application for acquiring further information. In order to promote collaboration between digital information in the cloud (virtual) and exhibition items on site (reality), and also to investigate the benefits of applying $A R$ technologies to designing digital tours for museum artefacts. The content that reveal on the screen by scanning specific items is organised as an add-on virtual exhibition. This collaborative mechanism enforces a simple cementing of information between reality exhibition and virtual one.

The concept of design idea is illustrated as the following: 


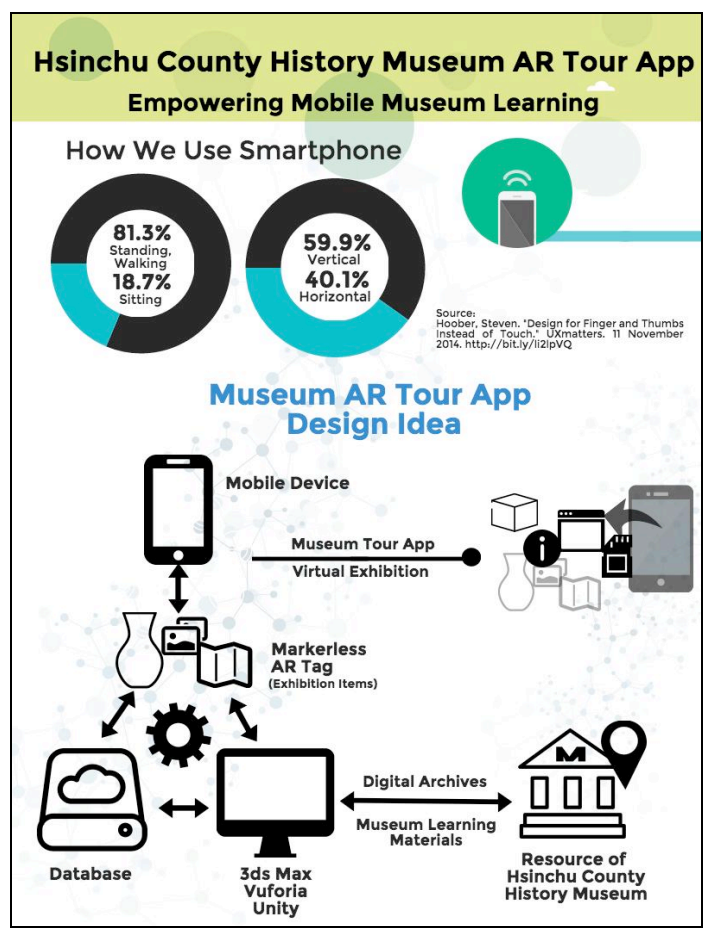

Figure 8. Museum AR Tour App Design Idea

\subsection{Application development}

The add-on exhibition doesn't reconstruct any existing installation and exhibition content, but serve as digitally popup screens, slides, audio, and animations added to the real exhibition artefacts with more interactive information instead. In order to achieve this design concept, the application must be able to interpret 27 designated exhibition items as markerless AR image / object and then correspondingly create virtual image overlaying on the mobile device's screen according to the angles and distance the mobile device is away from the AR "marker". Furthermore, this application is developed as the museum-dedicated software; therefore the application's UI has to meet the needs of making museum visits more convenient and interesting.

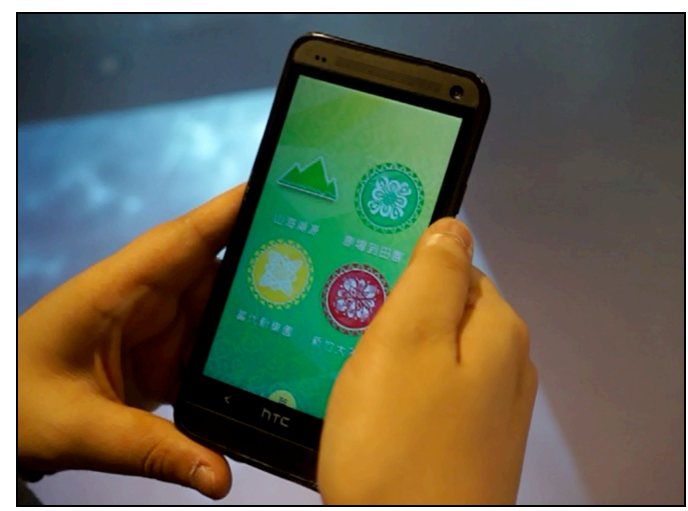

Figure 9. The UI is designed with vivid colour and icons

According to studies of Sung, Chang, \& Yu (2006), Tost \& Economou (2009), Wrzesien \& Raya (2010), poorly designed interactive device may have the potential risk of interference with visitors to learn from the exhibition. Nielsen (1993) proposed the quality of interface design could be assessed in two ways: usability and utility. There is 5 criteria can be used to evaluate the quality of usability: "Learnability", "Efficiency", "Memorability", "Errors", and "Satisfaction". As to the assessment of utility quality, the content of interface design is the core evaluation criteria. Furthermore, $\mathrm{Xu}$ (2010) and Kato et al. (2000) also indicate that the real attraction of augmented reality is its interactivity, ease of using, and pleasure.

For developing the application, we selected 27 items from the exhibition. These 27 items were analyzed and further categorized into 4 types according to their positions, contents, representative, and presentations. Considering the core feature of this museum tour application, the selected items were studied to assess their values for game-based learning purpose respectively. The AR function is used to present the supplementary information for the individual exhibition item. The supplementary information is extracted from historical materials that are preserved in the reference room, and to be presented in form of virtual AR objects by scanning the relevant exhibition item.

\subsection{Result}

To seek proper usability and utility of AR supplementary information, the virtual AR imaging is displayed under comprehensive consideration and in forms of video, animation, game, 3D object, and graphic material. By applying markerless AR SDK to enable device camera to scan and calculate the size of exhibition objects and then to accurately overlay them with virtual imaging. These 27 selected items, which are distributed in 4 independent exhibition areas, can serve as personal museum tour guide to create a pleasant and engaging visitor experience. Without the restrictions regarding the location and device, or even a standard method of control, markerless AR enables individual museum tour anywhere and any time by simply transforming a real exhibition room into

In this case, application of AR museum tour can help rendering a more tangible presentation about the artefacts of Hsinchu County History Museum. The mobile guiding application allows visitors to freely explore, learn and research in the museum. Research also shows that museum guides developed using AR technology often increase visitors' curiosity and interests as well as higher interactivity with the museum tour. In addition, AR museum tour application not only creates more interactive environment, but also reduces the cost for creating such environment.

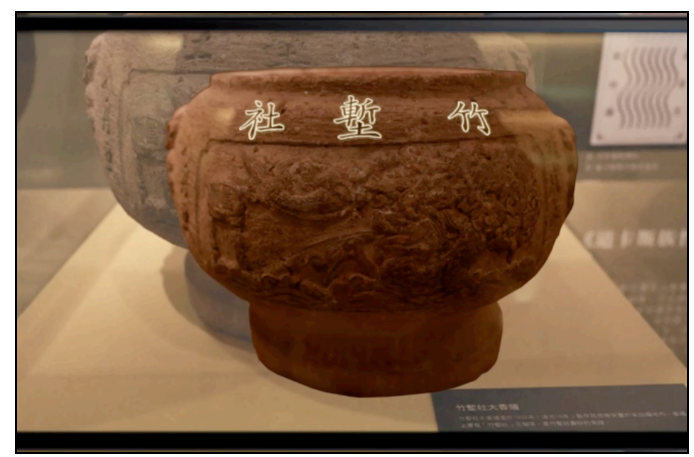

Figure 10. Scanning corroded item to show its original appearance, which is recovered from the historical archive. 


\section{CONCLUSION}

In this study, two prototype applications with different interaction styles and development purposes were constructed. The attention to the historical artefacts of the Hukou Old Street and Hsinchu County History Museum is strengthened as expected. These results encouraged The Digital ARt / ARchitecture Project to develop more applications / games to meet the needs of applying mobile device as the interactive learning tool. Even though learning through AR application is interesting, there are some issues deserve further studies. First, due to $3 \mathrm{D}$ models are necessary, specific algorithms for realtime rendering of $3 \mathrm{D}$ models for $\mathrm{AR}$ should be developed. Second, since most mobile devices use the touch screen as the input and output user interfaces, he mapping should be natural and intuitive to help users concentrate on the digital contents.

The present project enables players, or visitors especially in this project, to bring magnificent architectures and historical artefacts closer to see and use for interactive learning. The solution of this project makes cultural and historical materials portable and interactive, and furthermore, to transform users into players to interact with $\mathrm{AR}$ content in an intuitive and exciting manner.

For presenting AR component in a more attractive and functional way, future works will integrate the AR target into the form and function of publications that are more appealing and suitable for user to absorb educational information and appreciate $3 \mathrm{D}$ virtual objects simultaneously. It means the transformation of AR target markers will also function as essential approach of production development to accomplish both business and educational purposes.

\section{REFERENCES}

Azuma, R. T., 1997. A survey of augmented reality. Presence: Teleoperators and Virtual Environments, 6(4), pp. 355-385.

Billinghurst, M., Grasset, R., \& Seichter, H., 2010. Tangible interfaces for ambient augmented reality applications. In: Human-Centric Interfaces for Ambient Intelligence. Elsevier, New York, pp. 281-298.

Carrozzino, M., Evangelista, C., Scucces, A., Tecchia, F., Tennirelli, G., \& Bergamasco,

Chen, C.-H., Hsu, M.-F., Lee, I.-J., Sun, K.-W., Lin, Y.-C., \& Lee, W.-H., 2009. Applying augmented reality to visualize the history of traditional architecture in Taiwan. Paper presented at the Proceedings of 22nd CIPA.

Dade Robertson, M., 2009. Supporting the creation of hybrid museum experiences. In: Proceedings of the 27th International Conference on Human Factors in Computing Systems, pp. 1973-1982.

Franz Lehner, H. N., 2002. The role of mobile devices in elearning $\sim$ first experiences with a wireless e-learning environment.

Foni, A. E., Papagiannakis, G., \& Magnenat-Thalmann, N., 2010. A taxonomy of visualization strategies for cultural heritage applications. Journal of Computing and Cultural Heritage (JOCCH), 3(1), pp. 1-21.
Grasset, R., Dünser, A., \& Billinghurst, M., 2008. Edutainment with a mixed reality book: A visually augmented illustrative childrens' book. In: Proceedings of the 2008 International Conference on Advances in Computer Entertainment Technology, pp. 292-295.

Henderson, S., \& Feiner, S., 2010. Opportunistic tangible user interfaces for augmented reality. IEEE Transactions on Visualization and Computer Graphics, 16(1), pp. 4-16.

Kato, H., Billinghurst, M., Poupyrev, I., Imamoto, K., \& Tachibana, K., 2000. Virtual object manipulation on a table-top AR environment. In: Proceedings of the IEEE and ACM International Symposium on Augmented Reality (ISAR 2000), pp. 111-119.

Kuo, C.-C., Jeng, T.-S., 2008. The research of augmented reality registration technology applying on architecture and urban outdoor guiding systems using "cultural heritage guiding system" and "invisible shop signboard" as examples. Journal of Architecture, 66(12), pp.145-166.

Sung, Y.-T., Chang, K.-E., Yu, W.-J., 2006. Usage of mobile devices in museums: Promotion of human-computer-situation interactive design. Museology Quarterly, 20(1), pp.17-34.

Tost, L. P., Economou, M., 2007. Exploring the suitability of virtual reality interactivity for exhibitions through an integrated evaluation: The case of the Ename Museum. Online International Museology Journal, 4, pp. 81-97.

Wrzesien, M., Raya, M. A., 2010. Learning in serious virtual worlds: Evaluation of learning effectiveness and appeal to students in the e-junior project. Journal of Computers and Education, 55(1), pp.178-187. 